\title{
Hölder norm estimate for a Hilbert transform in Hermitean Clifford analysis
}

\author{
R. Abreu-Blaya*, J. Bory-Reyes ${ }^{\dagger}$, F. Brackx ${ }^{\ddagger}$, H. De Schepper ${ }^{\ddagger}$, F. Sommen ${ }^{\ddagger}$ \\ * Facultad de Informática y Matemática, Universidad de Holguín, Cuba \\ $\dagger$ Departamento de Matemática, Universidad de Oriente, Cuba \\ $\ddagger$ Clifford Research Group, Faculty of Engineering, Ghent University, Belgium
}

\begin{abstract}
A Hilbert transform for Hölder continuous circulant $(2 \times 2)$ matrix functions, on the $d$ summable (or fractal) boundary $\Gamma$ of a Jordan domain $\Omega$ in $\mathbb{R}^{2 n}$, has recently been introduced within the framework of Hermitean Clifford analysis. The main goal of the present paper is to estimate the Hölder norm of this Hermitean Hilbert transform. The expression for the upper bound of this norm is given in terms of the Hölder exponents, the diameter of $\Gamma$ and a specific $\mathbf{d}$-sum $(\mathbf{d}>d)$ of the Whitney decomposition of $\Omega$. The result is shown to include a more standard Hilbert transform for domains with left Ahlfors-David regular boundary.
\end{abstract}

Keywords: Hermitean Clifford analysis, Hilbert transform, fractal geometry.

Mathematics Subject Classification (2000): 30G35.

\section{Introduction}

In signal analysis the Hilbert transform of a real time signal is a fundamental tool. In particular the related notion of analytic signal is at the basis of various methods. If $f(x) \in L_{2}(\mathbb{R})$ and $H[f]$ denotes its Hilbert transform given by

$$
H[f](x)=\operatorname{Pv} \int_{-\infty}^{+\infty} \frac{f(y)}{x-y} d y
$$

then the corresponding analytic signal $\frac{1}{2} f+\frac{i}{2} H[f]$ belongs to the Hardy space $H^{2}(\mathbb{R})$ and arises as the $L_{2}$ nontangential boundary limit of the holomorphic Cauchy integral of $f$ in the upper half of the complex plane. The multidimensional approach to the Hilbert transform of a suitable function $f$ usually is tensorial, considering the Riesz transforms in each of the Cartesian variables separately, i.e.

$$
R_{j}[f](x)=\lim _{\varepsilon \rightarrow 0+} \frac{2}{a_{m+1}} \int_{\mathbb{R}^{m} \backslash B(x, \varepsilon)} \frac{x_{j}-y_{j}}{|x-y|^{m+1}} f(y) d V(y), \quad j=1, \ldots, m
$$

where $a_{m+1}$ denotes the area of the unit sphere $S^{m}$ in $\mathbb{R}^{m+1}$.

As opposed to this, Clifford analysis allows for a treatment of multidimensional phenomena, encompassing all dimensions at once. Clifford analysis is a comprehensive function theory offering a generalization to higher dimension of holomorphic function theory in the complex plane, see e.g. $[12,21,26,17,25]$. It focusses on monogenic functions, i.e. null solutions of the Dirac operator $\partial_{\underline{X}}=\sum_{j=1}^{m} e_{j} X_{j}$, where $\left(e_{1}, \ldots, e_{m}\right)$ is an orthogonal basis for $\mathbb{R}^{m}$, underlying the construction of the real Clifford algebra $\mathbb{R}_{0, m}$. Clifford analysis may also be considered as a refinement of harmonic analysis, since, as does the Cauchy-Riemann operator in the complex plane, the rotation-invariant Dirac operator factorizes the Laplacian. The theory of Hardy spaces in Clifford analysis is by now well established, see [16, 35, 19, 7], and the multidimensional Hilbert transform, as well as more general singular integral operators have been studied intensively, see $[28,26,35,39,29,18,20]$, in particular on Lipschitz hypersurfaces, see [32, 31, 34], and on smooth closed hypersurfaces, such as the unit sphere, see [19, 13]. 
In $\mathbb{R}^{2 n} \cong \mathbb{C}^{n}$, Hermitean Clifford analysis has emerged as yet a refinement of the Euclidean setting. Here, Hermitean monogenic functions are considered, i.e. functions taking values in the complex Clifford algebra $\mathbb{C}_{2 n}$ and being simultaneous null solutions of two complex Hermitean Dirac operators, which are invariant under the action of the unitary group. The study of complex Dirac operators (also in other settings) was initiated in [37, 36, 38]; however, a systematic development of the Hermitean function theory is still in full progress, see e.g. [15, 8, 9, 14].

In [10] a Hermitean Hilbert transform is introduced as a part of the non-tangential boundary limit of the Hermitean Cauchy integral constructed in [11] for domains with $C^{\infty}$ smooth boundaries, using a matrix approach with circulant $(2 \times 2)$ matrix functions. More recently, see [3], the above operators were redefined for Ahlfors-David surfaces and applied to solve boundary value problems for Hermitean monogenic matrix functions, see [5]. The case of fractal boundaries, however, is not covered by the method developed there. This is the reason why in $[2,4,1]$ the authors introduce an alternative way of defining the Hermitean matrix Hilbert transform over a fractal surface. As a consequence, also some results of [3] are extended to this more general context.

The Hilbert transform on the real line is an isometry on the space $L_{2}(\mathbb{R})$, and likewise, the corresponding $m$-dimensional Clifford-Hilbert transform has been shown to be an isometry on $L_{2}\left(\mathbb{R}^{m}\right)$. Looking at generalizations of the Hilbert transform, the resulting operators usually still are bounded on the appropriate classes of functions, see $[13,10]$. The main goal of the present paper is to investigate this property for the Hermitean Hilbert transform for Hölder continuous circulant $(2 \times 2)$ matrix functions defined on the $d$-summable boundary $\Gamma$ of a Jordan domain $\Omega$ in $\mathbb{R}^{2 n}$. More precisely, an estimate will be established for its Hölder norm, in terms of the Hölder exponents, the diameter of $\Gamma$ and a specific $\mathbf{d}$-sum $(\mathbf{d}>d)$ of the Whitney decomposition of $\Omega$.

\section{Preliminaries}

\subsection{The Hermitean Clifford analysis setting}

Let $\left(e_{1}, \ldots, e_{m}\right)$ be an orthonormal basis of Euclidean space $\mathbb{R}^{m}$ and consider the complex Clifford algebra $\mathbb{C}_{m}$ constructed over $\mathbb{R}^{m}$, with a non-commutative multiplication, governed by the rules

$$
e_{j}^{2}=-1 \text { and } e_{j} e_{k}+e_{k} e_{j}=0, \quad j, k=1, \ldots, m, j \neq k
$$

and $\mathbb{C}_{m}$ is then generated additively by the elements $e_{A}=e_{j_{1}} \ldots e_{j_{k}}$, for $A=\left\{j_{1}, \ldots, j_{k}\right\} \subset$ $\{1, \ldots, m\}$ with $j_{1}<\cdots<j_{k}$, and $e_{\emptyset}=1$, for $A=\emptyset$. Any Clifford number $\lambda \in \mathbb{C}_{m}$ may thus be written as $\lambda=\sum_{A} \lambda_{A} e_{A}, \lambda_{A} \in \mathbb{C}$, its Hermitean conjugate $\lambda^{\dagger}$ being defined by $\lambda^{\dagger}=\sum_{A} \lambda_{A}^{c} \bar{e}_{A}$, where the bar denotes the real Clifford algebra conjugation, i.e. the main anti-involution for which $\bar{e}_{j}=-e_{j}$, and $\lambda_{A}^{c}$ stands for the complex conjugate of $\lambda_{A}$. Euclidean space $\mathbb{R}^{m}$ is embedded in the Clifford algebra $\mathbb{C}_{m}$ by identifying $\left(x_{1}, \ldots, x_{m}\right)$ with the real Clifford vector $\underline{X}$ given by $\underline{X}=\sum_{j=1}^{m} e_{j} x_{j}$, for which $\underline{X}^{2}=-<\underline{X}, \underline{X}>=-|\underline{X}|^{2}$. The Fischer dual of $\underline{X}$ is the vector valued first order Dirac operator $\partial_{\underline{X}}=\sum_{j=1}^{m} e_{j} \partial_{x_{j}}$, factorizing the Laplacian: $\Delta_{m}=-\partial_{\underline{X}}^{2}$; it underlies the notion of monogenicity of a function: a continuously differentiable function $g$, defined in an open region $\Omega$ of $\mathbb{R}^{m}$, and taking values in $\mathbb{C}_{m}$, is called (left) monogenic in $\Omega$ iff $\partial_{\underline{X}} g=0$ in $\Omega$.

The transition to the Hermitean Clifford setting, see e.g. [8, 15], is essentially based on the introduction of a complex structure $J$, i.e. an $\mathrm{SO}(m)$ element for which $J^{2}=-\mathbf{1}_{m}$. This forces the dimension of the vector space to be even, whence we put $m=2 n$ from now on. A possible realization of the complex structure then is $J\left[e_{2 j-1}\right]=-e_{2 j}$ and $J\left[e_{2 j}\right]=e_{2 j-1}, j=1, \ldots, n$. The real Clifford vector and its corresponding Dirac operator are now denoted as

$$
\underline{X}=\sum_{j=1}^{n}\left(e_{2 j-1} x_{2 j-1}+e_{2 j} x_{2 j}\right), \quad \partial_{\underline{X}}=\sum_{j=1}^{n}\left(e_{2 j-1} \partial_{x_{2 j-1}}+e_{2 j} \partial_{x_{2 j}}\right)
$$

while we will also consider their counterparts obtained through the action of $J$, i.e.

$$
\underline{X} \mid=J[\underline{X}]=\sum_{j=1}^{n}\left(e_{2 j-1} x_{2 j}-e_{2 j} x_{2 j-1}\right), \quad \partial_{\underline{X} \mid}=J\left[\partial_{\underline{X}}\right]=\sum_{j=1}^{n}\left(e_{2 j-1} \partial_{x_{2 j}}-e_{2 j} \partial_{x_{2 j-1}}\right)
$$

As was the case with $\partial_{\underline{X}}$, a notion of monogenicity may be associated to $\partial_{\underline{X} \mid}$ as well. 
The Hermitean Clifford variables are then given by $\underline{Z}=\frac{1}{2}(\underline{X}+i \underline{X} \mid)$ and $\underline{Z}^{\dagger}=-\frac{1}{2}(\underline{X}-i \underline{X} \mid)$, while the Hermitean Dirac operators are defined as $\partial_{\underline{Z}}=-\frac{1}{4}\left(\partial_{\underline{X}}-i \partial_{\underline{X}}\right)$ and $\bar{\partial}_{\underline{Z}^{\dagger}}=\frac{1}{4}\left(\partial_{\underline{X}}+i \partial_{\underline{X}}\right)$. The Hermitean vector variables and Dirac operators are isotropic, i.e. $(\underline{Z})^{2}=\left(\underline{Z}^{\dagger}\right)^{2}=0$ and $\left(\partial_{\underline{Z}}\right)^{2}=\left(\partial_{Z^{\dagger}}\right)^{2}=0$, whence the Laplacian allows for the decomposition $\Delta_{2 n}=4\left(\partial_{\underline{Z}} \partial_{Z^{\dagger}}+\partial_{Z^{\dagger}} \partial_{\underline{Z}}\right)$. These objects lie at the core of the Hermitean function theory by means of the following definition.

Definition 1 A continuously differentiable function $g$ in $\Omega \subset \mathbb{R}^{2 n}$ with values in $\mathbb{C}_{2 n}$ is called left Hermitean monogenic (or left h-monogenic) in $\Omega$, iff it satisfies the system $\partial_{\underline{Z}} g=0=\partial_{\underline{Z}^{\dagger}} g$ or the equivalent system $\partial_{\underline{X}} g=0=\partial_{\underline{X} \mid} g$.

Right h-monogenicity is defined similarly. Functions which are both left and right h-monogenic are called two-sided h-monogenic. The above definition constitutes a refinement of monogenicity, since h-monogenic functions (left or right) are monogenic w.r.t. both Dirac operators $\partial_{\underline{X}}$ and $\partial_{\underline{X}}$.

\subsection{The transition to a circulant matrix approach}

The fundamental solutions of the Dirac operators $\partial_{\underline{X}}$ and $\partial_{\underline{X}}$ are respectively given by

$$
E(\underline{X})=-\frac{1}{a_{2 n}} \frac{\underline{X}}{|\underline{X}|^{2 n}}, \quad E \mid(\underline{X})=-\frac{1}{a_{2 n}} \frac{\underline{X} \mid}{|\underline{X}|^{2 n}}, \quad \underline{X} \in \mathbb{R}^{2 n} \backslash\{0\}
$$

where $a_{2 n}$ denotes the surface area of the unit sphere in $\mathbb{R}^{2 n}$. Introducing the functions

$$
\mathcal{E}(\underline{Z})=-(E+i E \mid)=\frac{2}{a_{2 n}} \frac{\underline{Z}}{|\underline{Z}|^{2 n}} \quad \text { and } \quad \mathcal{E}^{\dagger}(\underline{Z})=(E-i E \mid)=\frac{2}{a_{2 n}} \frac{\underline{Z}^{\dagger}}{|\underline{Z}|^{2 n}}
$$

as well as the particular circulant $(2 \times 2)$ matrices

$$
\mathcal{D}_{\left(\underline{Z}, \underline{Z}^{\dagger}\right)}=\left(\begin{array}{ll}
\partial_{\underline{Z}} & \partial_{\underline{Z}^{\dagger}} \\
\partial_{\underline{Z}^{\dagger}} & \partial_{\underline{Z}}
\end{array}\right), \quad \mathcal{E}=\left(\begin{array}{ll}
\mathcal{E} & \mathcal{E}^{\dagger} \\
\mathcal{E}^{\dagger} & \mathcal{E}
\end{array}\right) \quad \text { and } \quad \delta=\left(\begin{array}{cc}
\delta & 0 \\
0 & \delta
\end{array}\right)
$$

where $\delta$ is the Dirac delta distribution, one obtains that $\mathcal{D}_{\left(\underline{Z}, \underline{Z}^{\dagger}\right)} \mathcal{E}(\underline{Z})=\boldsymbol{\delta}(\underline{Z})$, so that $\mathcal{E}$ may be considered as a fundamental solution of $\mathcal{D}_{\left(\underline{Z}, \underline{Z}^{\dagger}\right)}$ in a matricial context, see e.g. [10, 11, 36]. Moreover, the Dirac matrix $\mathcal{D}_{\left(\underline{Z}, \underline{Z}^{\dagger}\right)}$ in some sense factorizes the Laplacian, since

$$
4 \mathcal{D}_{\left(\underline{Z}, \underline{Z}^{\dagger}\right)}\left(\mathcal{D}_{\left(\underline{Z}, \underline{Z}^{\dagger}\right)}\right)^{\dagger}=\left(\begin{array}{cc}
\Delta_{2 n} & 0 \\
0 & \Delta_{2 n}
\end{array}\right) \equiv \boldsymbol{\Delta}
$$

This observation inspired the following definition.

Definition 2 Let $g_{1}, g_{2}$ be continuously differentiable functions defined in $\Omega$ and taking values in $\mathbb{C}_{2 n}$, and consider the matrix function

$$
\boldsymbol{G}_{2}^{1}=\left(\begin{array}{cc}
g_{1} & g_{2} \\
g_{2} & g_{1}
\end{array}\right)
$$

Then $\boldsymbol{G}_{2}^{1}$ is called left (respectively right) $\mathbf{H}$-monogenic in $\Omega$ iff it satisfies in $\Omega$ the system $\mathcal{D}_{\left(\underline{Z}, \underline{Z}^{\dagger}\right)} \boldsymbol{G}_{2}^{1}=\boldsymbol{O}$ (respectively $\boldsymbol{G}_{2}^{1} \mathcal{D}_{\left(\underline{Z}, \underline{Z}^{\dagger}\right)}=\boldsymbol{O}$ ). Here $\boldsymbol{O}$ denotes the matrix with zero entries.

Notions of continuity, differentiability and integrability of $\boldsymbol{G}_{2}^{1}$ are introduced entry-wise. In particular, we consider the classes $\mathcal{C}^{0, \alpha}(\mathbf{E})$ and $\boldsymbol{L}_{\boldsymbol{p}}(\mathbf{E})$ of, respectively, Hölder continuous and $p$ integrable circulant matrix functions over some suitable subset $\mathbf{E}$ of $\mathbb{R}^{2 n}$. However, introducing the non-negative function $\left\|\boldsymbol{G}_{2}^{1}(\underline{X})\right\|=\max \left\{\left|g_{1}(\underline{X})\right|,\left|g_{2}(\underline{X})\right|\right\}$, these function classes may also be defined by means of the traditional conditions

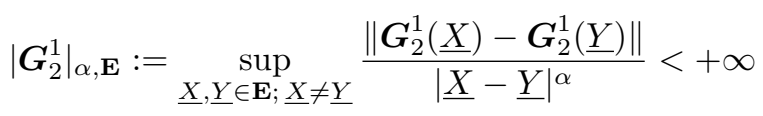

and

$$
\left\|G_{2}^{1}\right\|_{L_{p}}:=\left(\int_{\mathbf{E}}\left\|G_{2}^{1}(\underline{X})\right\|^{p}\right)^{\frac{1}{p}}<+\infty
$$

respectively. The non-negative numbers $\left\|\boldsymbol{G}_{2}^{1}\right\|_{\alpha, \mathbf{E}}:=\max _{\underline{X} \in \mathbf{E}}\left\|\boldsymbol{G}_{2}^{1}(\underline{X})\right\|+\left|\boldsymbol{G}_{2}^{1}\right|_{\alpha, \mathbf{E}}$ and $\left\|\boldsymbol{G}_{2}^{1}\right\|_{L_{p}}$ are then said to be the norms of an element $\boldsymbol{G}_{2}^{1}$ in $\mathcal{C}^{0, \alpha}(\mathbf{E})$ or $\boldsymbol{L}_{\boldsymbol{p}}(\mathbf{E})$, respectively.

From now on we denote by $c, c_{1}, c_{2}, c_{\Gamma}, \ldots$ generic positive constants, which only depend on the parameters which are explicitly indicated and can take different values. 


\subsection{Box dimension and $d$-summable sets in $\mathbb{R}^{2 n}$}

Let $\mathbf{E}$ be an arbitrary bounded subset of $\mathbb{R}^{2 n}$, whose diameter will be denoted by $|\mathbf{E}|$. Then for any $s \geq 0$ its Hausdorff measure $\mathcal{H}^{s}(\mathbf{E})$ may be defined by

$$
\mathcal{H}^{s}(\mathbf{E})=\lim _{\delta \rightarrow 0} \inf \left\{\sum_{k=1}^{\infty}\left|B_{k}\right|^{s}: \mathbf{E} \subset \bigcup_{k=1}^{\infty} B_{k},\left|B_{k}\right|<\delta\right\}
$$

the infimum being taken over all countable $\delta$-coverings $\left\{B_{k}\right\}$ of $\mathbf{E}$ with open or closed balls. For $s=2 n$, the Hausdorff measure $\mathcal{H}^{2 n}$ coincides, up to a positive multiplicative constant, with the Lebesgue measure $\mathcal{L}^{2 n}$ in $\mathbb{R}^{2 n}$.

Now, let $\mathbf{E}$ be compact. The Hausdorff dimension $\alpha_{H}(\mathbf{E})$ of $\mathbf{E}$ is defined as the infimum of all $s \geq 0$ such that $\mathcal{H}^{s}(\mathbf{E})<+\infty$. For more details concerning the Hausdorff measure and dimension we refer to [22, 23]. Frequently however, see [30], the so-called box dimension is used, defined as

$$
\alpha(\mathbf{E})=\lim _{\varepsilon \rightarrow 0} \sup \frac{\log N_{\mathbf{E}}(\varepsilon)}{-\log \varepsilon}
$$

where $\mathbf{N}_{\mathbf{E}}(\varepsilon)$ stands for the minimal number of $\varepsilon$-balls needed to cover $\mathbf{E}$. Note that the limit above remains unchanged if $\mathbf{N}_{\mathbf{E}}(\varepsilon)$ is replaced by the number of $k$-cubes intersecting $\mathbf{E}$, with $2^{-k} \leq \varepsilon<$ $2^{-k+1}$. A cube is called a $k$-cube if it is of the form $\left[l_{1} 2^{-k},\left(l_{1}+1\right) 2^{-k}\right] \times \cdots \times\left[l_{2 n} 2^{-k},\left(l_{2 n}+1\right) 2^{-k}\right]$, where $k$ and $l_{1}, \ldots, l_{2 n}$ are integers. The box dimension and the Hausdorff dimension of a given compact set $\mathbf{E}$ can be equal, which is for instance the case for the so-called $(2 n-1)$-rectifiable sets, see [24], but in general we have that $\alpha_{H}(\mathbf{E}) \leq \alpha(\mathbf{E})$. The following geometric notion was introduced in [27], and is essential in their method of integrating a form over a fractal boundary.

Definition 3 The compact set $\mathbf{E}$ is said to be d-summable iff the improper integral $\int_{0}^{1} N_{\mathbf{E}}(\tau) \tau^{d-1} d \tau$ converges.

\section{Lemma 1 It holds that}

(i) any d-summable set $\mathbf{E}$ has box dimension $\alpha(\mathbf{E}) \leq d$;

(ii) if $\alpha(\mathbf{E})<d$, then $\mathbf{E}$ is d-summable;

(iii) if $\mathbf{E}$ is $d$-summable, then it is also $(d+\varepsilon)$-summable for every $\varepsilon>0$.

In what follows, we will take $\Omega \subset \mathbb{R}^{2 n}$ to be a Jordan domain, i.e. a bounded oriented connected open subset of $\mathbb{R}^{2 n}$, the boundary $\Gamma$ of which is a compact topological surface. For our purpose, we will assume that the Hausdorff and box dimensions of $\Gamma$ satisfy $2 n-1 \leq \alpha_{H}(\Gamma) \leq \alpha(\Gamma)<2 n$. Note that this includes the case when $\Gamma$ is fractal in the sense of Mandelbrot, i.e. when $2 n-1<\alpha_{H}(\Gamma)$. Under these conditions, there will always exist $d \in[2 n-1,2 n[$ such that $\Gamma$ is $d$-summable, on account of Lemma 1.

We will also need the so-called Whitney decomposition of $\Omega$, which we will only recall briefly; for details we refer to [40]. Consider the lattice $\mathbb{Z}^{2 n}$ in $\mathbb{R}^{2 n}$ and the collection of closed unit cubes defined by it; let $\mathcal{M}_{1}$ be the mesh consisting of those unit cubes having a non-empty intersection with $\Omega$. We then recursively define the meshes $\mathcal{M}_{k}, k=2,3, \ldots$, each time bisecting the sides of the cubes of the previous one. The cubes in $\mathcal{M}_{k}$ thus have side length $2^{-k+1}$ and diameter $|Q|=\sqrt{2 n} 2^{-k+1}$. We then define, for $k=2,3, \ldots$,

$$
\begin{aligned}
& \mathcal{W}^{1}=\left\{Q \in \mathcal{M}_{1} \mid \text { all neighbour cubes of } Q \text { belong to } \Omega\right\} \\
& \mathcal{W}^{k}=\left\{Q \in \mathcal{M}_{k} \mid \text { all neighbour cubes of } Q \text { belong to } \Omega, \text { and } \nexists Q^{*} \in \mathcal{W}^{k-1}: Q \subset Q^{*}\right\}
\end{aligned}
$$

for which it can be proven that $\Omega=\bigcup_{k=1}^{+\infty} \mathcal{W}^{k}=\bigcup_{k=1}^{+\infty} \bigcup_{Q \in \mathcal{W}^{k}} Q \equiv \bigcup_{Q \in \mathcal{W}} Q$, all cubes $Q$ in the Whitney decomposition $\mathcal{W}$ of $\Omega$ having disjoint interiors. We then have the following relation between the $d$-summability of the boundary $\Gamma$ and the Whitney decomposition of $\Omega$, see [27].

Lemma 2 If $\Omega$ is a Jordan domain of $\mathbb{R}^{2 n}$ and its boundary $\Gamma$ is d-summable, then the expression $\mathbf{s}(d)=\sum_{Q \in \mathcal{W}}|Q|^{d}$, called the d-sum of the Whitney decomposition $\mathcal{W}$ of $\Omega$, is finite. 
An important special case is when $\Gamma$ is assumed to be left Ahlfors-David regular, or $(\ell)$ ADregular for short, meaning that $\mathcal{H}^{2 n-1}(\Gamma)<\infty$, and there exists a constant $c_{\Gamma}$ such that

$$
c_{\Gamma} r^{2 n-1} \leq \mathcal{H}^{2 n-1}(\Gamma \cap B(\underline{U}, r)) \text { for all } \underline{U} \in \Gamma \text { and } 0<r \leq|\Gamma|
$$

where $B(\underline{U}, r)$ denotes the closed ball with center $\underline{U}$ and radius $r$. A nice link between this geometric notion, introduced in [6], and the one of $d$-summability is given in the following lemma.

Lemma 3 If $\Gamma$ is $(\ell)$ AD-regular, then $\Gamma$ is $(2 n-1+\varepsilon)$-summable for all $\varepsilon>0$. Moreover,

$$
\mathbf{s}(d) \leq c \frac{\mathcal{H}^{2 n-1}(\Gamma)}{\varepsilon}
$$

Proof.

The $(2 n-1+\varepsilon)$-summability of $\Gamma$ is obtained by observing that $N_{\Gamma}(\tau) \leq P_{\Gamma}\left(\frac{\tau}{2}\right)$, where $P_{\Gamma}\left(\frac{\tau}{2}\right)$ is the packing number of $\Gamma$, i.e. the biggest number of disjoint $\frac{\tau}{2}$ balls with center in $\Gamma$, see [33]. Then, as $\Gamma$ is $(\ell)$ AD-regular, we have

$$
c_{\Gamma} \frac{\tau^{2 n-1}}{2^{2 n-1}} P_{\Gamma}\left(\frac{\tau}{2}\right) \leq \mathcal{H}^{2 n-1}(\Gamma)
$$

and hence $N_{\Gamma}(\tau) \leq c \mathcal{H}^{2 n-1}(\Gamma) \tau^{1-2 n}$, where the constant $c$ depends on $n$ and $c_{\Gamma}$. The proof now proceeds similarly to the one of [27, Lemma 2$]$.

\section{A Hermitean Hilbert transform on $d$-summable surfaces}

For further use, we introduce the notations $\Omega^{+} \equiv \Omega$, and $\Omega^{-} \equiv \mathbb{R}^{2 n} \backslash \bar{\Omega}$. From now on we reserve the notations $\underline{Y}$ and $\underline{Y} \mid$, respectively $\underline{U}$ and $\underline{U} \mid$ for Clifford vectors associated to points in $\mathbb{R}^{2 n} \backslash \Gamma$, while their Hermitean counterparts are denoted by $\underline{V}=\frac{1}{2}(\underline{Y}+i \underline{Y} \mid)$ and $\underline{V}^{\dagger}=-\frac{1}{2}(\underline{Y}-i \underline{Y} \mid)$. Similarly, the notations $\underline{U}$ and $\underline{U} \mid$ mean Clifford vectors associated to points in $\Gamma$, while their Hermitean counterparts are denoted by $\underline{W}=\frac{1}{2}(\underline{U}+i \underline{U} \mid)$ and $\underline{W}^{\dagger}=-\frac{1}{2}(\underline{U}-i \underline{U} \mid)$.

Assuming that $\mathcal{H}^{2 n-1}(\Gamma)<\infty$, the Hermitean Cauchy integral of a matrix function $\boldsymbol{G}_{2}^{1} \in$ $\mathcal{C}^{0, \alpha}(\Gamma)$ is defined by

$$
\mathcal{C}_{\Gamma} \boldsymbol{G}_{2}^{1}(\underline{Y})=\int_{\Gamma} \mathcal{E}(\underline{Z}-\underline{V}) \mathbf{N}_{\left(\underline{Z}, \underline{Z}^{\dagger}\right)} \boldsymbol{G}_{2}^{1}(\underline{X}) d \mathcal{H}^{2 n-1}, \quad \underline{Y} \in \Omega^{ \pm}
$$

where the circulant matrix

$$
\mathbf{N}_{\left(\underline{Z}, \underline{Z}^{\dagger}\right)}=\left(\begin{array}{cc}
\underline{N} & -\underline{N}^{\dagger} \\
-\underline{N}^{\dagger} & \underline{N}
\end{array}\right)
$$

contains (up to a constant factor) the Hermitean projections $\underline{N}$ and $\underline{N}^{\dagger}$ of the unit normal vector $\underline{n}(\underline{U})$ at $\underline{U} \in \Gamma$.

In [2], an alternative approach for defining the Hermitean Cauchy integral is presented, in the case of domains with $d$-summable boundary, by means of the following definition.

Definition 4 Let $\Omega$ be a Jordan domain in $\mathbb{R}^{2 n}$ with d-summable boundary $\Gamma, d \in[2 n-1,2 n[$. Moreover, let $d-2 n+1<\alpha \leq 1$ and consider $\boldsymbol{G}_{2}^{1} \in \mathcal{C}^{0, \alpha}(\Gamma)$. Then, for $\underline{Y} \in \mathbb{R}^{2 n} \backslash \Gamma$ the Hermitean Cauchy integral of $\boldsymbol{G}_{2}^{1}$ is defined by

$$
\left(\mathcal{C}_{\Gamma}^{*} \boldsymbol{G}_{2}^{1}\right)(\underline{Y})=(-1)^{\frac{n(n+1)}{2}}(2 i)^{n} \boldsymbol{\chi}_{\Omega}(\underline{Y}) \widetilde{\boldsymbol{G}}_{2}^{1}(\underline{Y})-\mathcal{T}_{\Omega} \mathcal{D}_{\left(\underline{Z}, \underline{Z}^{\dagger}\right.} \widetilde{\boldsymbol{G}}_{2}^{1}(\underline{Y})
$$

$\chi_{\Omega}$ being the diagonal matrix version of the standard characteristic function $\chi_{\Omega}$ of $\Omega$. Furthermore, $\mathcal{T}_{\Omega}$ denotes the Hermitean Téodorescu transform, given for $\boldsymbol{F}_{2}^{1} \in \mathcal{C}^{1}(\Omega)$ by

$$
\mathcal{T}_{\Omega} \boldsymbol{F}_{2}^{1}(\underline{Y})=-\int_{\Omega} \mathcal{E}(\underline{Z}-\underline{V}) \boldsymbol{F}_{2}^{1}(\underline{X}) d W\left(\underline{Z}, \underline{Z}^{\dagger}\right)
$$

where $d W\left(\underline{Z}, \underline{Z}^{\dagger}\right)$ is the associated volume element, given by

$$
d V(\underline{X})=(-1)^{\frac{n(n-1)}{2}}\left(\frac{i}{2}\right)^{n} d W\left(\underline{Z}, \underline{Z}^{\dagger}\right)
$$


reflecting integration in the respective underlying complex planes. Finally, $\widetilde{\boldsymbol{G}}_{2}^{1}$ denotes a Whitney type extension of $\boldsymbol{G}_{2}^{1}$, see [40], satisfying $\widetilde{\boldsymbol{G}}_{2}^{1} \in \mathcal{C}^{\infty}\left(\mathbb{R}^{2 n} \backslash \mathbf{E}\right),\left.\widetilde{\boldsymbol{G}}_{2}^{1}\right|_{\mathbf{E}}=\boldsymbol{G}_{2}^{1}$ and

$$
\left\|\mathcal{D}_{\left(\underline{Z}, \underline{Z}^{\dagger}\right)} \widetilde{\boldsymbol{G}}_{2}^{1}(\underline{Y})\right\| \leq c \operatorname{dist}(\underline{Y}, \mathbf{E})^{\alpha-1}, \quad \text { for all } \underline{Y} \in \mathbb{R}^{2 n} \backslash \mathbf{E}
$$

Direct verification shows that $\mathcal{C}_{\Gamma}^{*} \boldsymbol{G}_{2}^{1}$ is Hermitean monogenic in $R^{2 n} \backslash \Gamma$ and vanishes at infinity, while, in cases where $\Gamma$ is sufficiently regular, e.g. Ahlfors-David regular, the Hermitean Cauchy integral (2) reduces to the one considered in [3]. Furthermore, Definition 4 is legitimate, since the right hand side of (2) exists for any $\underline{Y} \in \mathbb{R}^{2 n} \backslash \Gamma$ and does not depend on the particular choice of the Whitney type extension $\widetilde{\boldsymbol{G}}_{2}^{1}$. A proof of this last assertion can be found in [2].

A natural question is whether $\mathcal{C}_{\Gamma}^{*} \boldsymbol{G}_{2}^{1}$ admits a continuous extension to $\bar{\Omega}=\Omega \cup \Gamma$, in which case a "fractal" Hermitean Hilbert transform could be introduced, respecting the traditional structure of the Plemelj-Sokhotski formulae:

$$
\mathcal{H}_{\Gamma}^{*} \boldsymbol{G}_{2}^{1}(\underline{U})=2 \frac{(-1)^{\frac{n(n+1)}{2}}}{(2 i)^{n}}\left(\mathcal{C}_{\Gamma}^{*} \boldsymbol{G}_{2}^{1}\right)^{+}(\underline{U})-\boldsymbol{G}_{2}^{1}(\underline{U}), \quad \underline{U} \in \Gamma
$$

where $\left(\mathcal{C}_{\Gamma}^{*} \boldsymbol{G}_{2}^{1}\right)^{+}$denotes the trace on $\Gamma$ of the continuous extension of $\mathcal{C}_{\Gamma}^{*} \boldsymbol{G}_{2}^{1}$ to $\bar{\Omega}$. Definition (3) would then provide an alternative for the matricial Hermitean Hilbert transform $\mathcal{H}$ introduced in [10] for domains with $C^{\infty}$ smooth boundaries. Under an additional condition on the regularity of the considered matrix function $\boldsymbol{G}_{2}^{1}$, this question indeed has been answered affirmatively, as stated in the following theorem proven in [1].

Theorem 1 Let $\Omega$ be a Jordan domain in $\mathbb{R}^{2 n}$ with $d$-summable boundary $\Gamma, d \in[2 n-1,2 n[$. Furthermore, let $d-2 n+1<\alpha \leq 1$ and consider $\boldsymbol{G}_{2}^{1} \in \mathcal{C}^{0, \alpha}(\Gamma)$. If moreover

$$
\alpha>\frac{d}{2 n}
$$

then $\mathcal{C}_{\Gamma}^{*} \boldsymbol{G}_{2}^{1}(\underline{Y})$ admits a continuous extension to $\bar{\Omega}$. Moreover, it then holds that the Hilbert transform $\mathcal{H}_{\Gamma}^{*} \boldsymbol{G}_{2}^{1}$, defined by (3), belongs to $\mathcal{C}^{0, \beta}(\Gamma)$, whenever $\beta<\frac{2 n \alpha-d}{2 n-d}<1$.

For the convenience of the reader we repeat the main ideas of the proof, thus making our exposition self-contained. Since $\alpha>\frac{d}{2 n}$ implies that $2 n<\frac{2 n-d}{1-\alpha}$, we may choose $p$ such that $2 n<p<\frac{2 n-d}{1-\alpha}$. For any such $p$, we have that $\mathcal{D}_{\left(\underline{Z}, \underline{Z}^{\dagger}\right)} \widetilde{\boldsymbol{G}}_{2}^{1} \in \boldsymbol{L}_{\boldsymbol{p}}(\Omega)$, so that the integral term $\mathcal{T}_{\Omega} \mathcal{D}_{\left(\underline{Z}, \underline{Z}^{\dagger}\right)} \widetilde{\boldsymbol{G}}_{2}^{1}(\underline{Y})$ in (2) represents a continuous function in $\mathbb{R}^{2 n}$. This clearly forces $\mathcal{C}_{\Gamma}^{*} \boldsymbol{G}_{2}^{1}(\underline{Y})$ to admit a continuous extension to $\bar{\Omega}$, whence $\mathcal{H}_{\Gamma}^{*} \boldsymbol{G}_{2}^{1}$ is well-defined. Moreover, $\boldsymbol{\mathcal { T }}_{\Omega} \mathcal{D}_{\left(\underline{Z}, \underline{Z}^{\dagger}\right)} \widetilde{\boldsymbol{G}}_{2}^{1}(\underline{Y}) \in \mathcal{C}^{0, \frac{p-2 n}{p}}\left(\mathbb{R}^{2 n}\right)$, which implies that $\mathcal{H}_{\Gamma}^{*} \boldsymbol{G}_{2}^{1} \in \mathcal{C}^{0, \beta}(\Gamma)$ for any $\beta$ satisfying $\beta<\frac{2 n \alpha-d}{2 n-d}$.

Remark 1 Observe that, still under condition (4), $\mathcal{H}_{\Gamma}^{*} \boldsymbol{G}_{2}^{1}$ may be rewritten as

$$
\mathcal{H}_{\Gamma}^{*} \boldsymbol{G}_{2}^{1}(\underline{U})=\boldsymbol{G}_{2}^{1}(\underline{U})-2 \frac{(-1)^{\frac{n(n+1)}{2}}}{(2 i)^{n}} \mathcal{T}_{\Omega} \mathcal{D}_{\left(\underline{Z}, \underline{Z}^{\dagger}\right)} \widetilde{\boldsymbol{G}}_{2}^{1}(\underline{U}),
$$

which is a natural generalization of the more conventional Hermitean Hilbert transform given by

$$
\left(\mathcal{H}_{\Gamma} \boldsymbol{G}_{2}^{1}\right)(\underline{U}):=\boldsymbol{G}_{2}^{1}(\underline{U})-2 \int_{\Gamma} \mathcal{E}(\underline{Z}-\underline{W}) \mathbf{N}_{\left(\underline{Z}, \underline{Z}^{\dagger}\right)}\left(\boldsymbol{G}_{2}^{1}(\underline{U})-\boldsymbol{G}_{2}^{1}(\underline{X}) d \mathcal{H}^{2 n-1}, \quad \underline{U} \in \Gamma .\right.
$$

\section{Hölder norm estimate for $\mathcal{H}_{\Gamma}^{*}$}

\subsection{The case of a $d$-summable boundary}

From Theorem 1 we learn that the Hilbert transform $\mathcal{H}_{\Gamma}^{*}$ acts from $\mathcal{C}^{0, \alpha}(\Gamma)$ into $\mathcal{C}^{0, \beta}(\Gamma)$ whenever

$$
0<\beta<\frac{2 n \alpha-d}{2 n-d}<1
$$

We will now show that $\mathcal{H}_{\Gamma}^{*}$ is a bounded operator between these spaces and present an upper bound for its Hölder norm. 
Theorem 2 Let $\Gamma$ be d-summable and suppose that condition (5) holds. Then $\mathcal{H}_{\Gamma}^{*}$ is bounded from $\mathcal{C}^{0, \alpha}(\Gamma)$ into $\mathcal{C}^{0, \beta}(\Gamma)$ and for its norm there holds that

$$
\left\|\boldsymbol{H}_{\Gamma}^{*}\right\| \leq 1+|\Gamma|^{\alpha-\beta}+c_{1}(\mathbf{s}(\mathbf{d}))^{\frac{1-\beta}{2 n}}+c_{2}(\mathbf{s}(\mathbf{d}))^{\frac{1-\beta}{2 n}}|\Gamma|^{\beta}
$$

where $\mathbf{d}=2 n \frac{\alpha-\beta}{1-\beta}$ and $c_{1}, c_{2}$ only depend on $\alpha, \beta$ and $n$.

Proof.

Choose $p=\frac{2 n}{1-\beta}$. The proof of Theorem 1 , see [1], then reveals that

$$
\int_{\Omega}\left\|\mathcal{D}_{\left(\underline{Z}, \underline{Z}^{\dagger}\right)} \widetilde{\boldsymbol{G}}_{2}^{1}(\underline{Y})\right\|^{p} \leq c\left|\boldsymbol{G}_{2}^{1}\right|_{\alpha, \Gamma}^{p} \sum_{Q \in \mathcal{W}}|Q|^{2 n-p(1-\alpha)}=c\left|\boldsymbol{G}_{2}^{1}\right|_{\alpha, \Gamma}^{p} \sum_{Q \in \mathcal{W}}|Q|^{p(\alpha-\beta)}=c\left|\boldsymbol{G}_{2}^{1}\right|_{\alpha, \Gamma}^{p} \mathbf{s}(\mathbf{d})
$$

where we have put $\mathbf{d}=2 n \frac{\alpha-\beta}{1-\beta}$. Since $\mathbf{d}>d$ and $\Gamma$ is $d$-summable, $\mathbf{s}(\mathbf{d})$ is finite and we have obtained

$$
\left\|\boldsymbol{D}_{\left(\underline{Z}, \underline{Z}^{\dagger}\right)} \widetilde{\boldsymbol{G}}_{2}^{1}\right\|_{L_{p}} \leq c^{1 / p}\left|\boldsymbol{G}_{2}^{1}\right|_{\alpha, \Gamma}(\mathbf{s}(\mathbf{d}))^{1 / p}
$$

At this stage we invoke [25, Proposition 8.1] to deduce that

$\left|\boldsymbol{\mathcal { T }}_{\Omega} \mathcal{D}_{\left(\underline{Z}, \underline{Z}^{\dagger}\right)} \widetilde{\boldsymbol{G}}_{2}^{1}(\underline{Y})\right| \leq c\left\|\mathcal{D}_{\left(\underline{Z}, \underline{Z}^{\dagger}\right)} \widetilde{\boldsymbol{G}}_{2}^{1}\right\|_{L_{p}}|\Gamma|^{\frac{p-2 n}{p}}=c\left\|\mathcal{D}_{\left(\underline{Z}, \underline{Z}^{\dagger}\right)} \widetilde{\boldsymbol{G}}_{2}^{1}\right\|_{L_{p}}|\Gamma|^{\beta} \leq c_{1}\left|\boldsymbol{G}_{2}^{1}\right|_{\alpha, \Gamma}(\mathbf{s}(\mathbf{d}))^{1 / p}|\Gamma|^{\beta}$ whence

$$
\left|\mathcal{T}_{\Omega} \mathcal{D}_{\left(\underline{Z}, \underline{Z}^{\dagger}\right)} \widetilde{\boldsymbol{G}}_{2}^{1}\right|_{\beta, \mathbb{R}^{2 n}} \leq c\left\|\mathcal{D}_{\left(\underline{Z}, \underline{Z}^{\dagger}\right)} \widetilde{\boldsymbol{G}}_{2}^{1}\right\|_{L_{p}} \leq c_{2}\left|\boldsymbol{G}_{2}^{1}\right|_{\alpha, \Gamma}(\mathbf{s}(\mathbf{d}))^{1 / p}
$$

Consequently, for any $\underline{U} \in \Gamma$, it holds that

$$
\begin{aligned}
\left|\mathcal{H}_{\Gamma}^{*} \boldsymbol{G}_{2}^{1}(\underline{U})\right| & \leq\left|\boldsymbol{G}_{2}^{1}(\underline{U})\right|+2\left|\mathcal{T}_{\Omega} \mathcal{D}_{\left(\underline{Z}, \underline{Z}^{\dagger}\right)} \widetilde{\boldsymbol{G}}_{2}^{1}(\underline{U})\right| \\
& \leq\left|\boldsymbol{G}_{2}^{1}(\underline{U})\right|+c_{1}\left|\boldsymbol{G}_{2}^{1}\right|_{\alpha, \Gamma}(\mathbf{s}(\mathbf{d}))^{1 / p}|\Gamma|^{\beta} \leq\left(1+c_{1}(\mathbf{s}(\mathbf{d}))^{1 / p}|\Gamma|^{\beta}\right)\left\|\boldsymbol{G}_{2}^{1}\right\|_{\alpha, \Gamma}
\end{aligned}
$$

whence

$$
\left|\mathcal{H}_{\Gamma}^{*} \boldsymbol{G}_{2}^{1}\right|_{\beta, \Gamma} \leq\left|\boldsymbol{G}_{2}^{1}\right|_{\beta, \Gamma}+2\left|\mathcal{T}_{\Omega} \mathcal{D}_{\left(\underline{Z}, \underline{Z}^{\dagger}\right)} \widetilde{\boldsymbol{G}}_{2}^{1}\right|_{\beta, \mathbb{R}^{2 n}} \leq\left(|\Gamma|^{\alpha-\beta}+c_{2}(\mathbf{s}(\mathbf{d}))^{1 / p}\right)\left|\boldsymbol{G}_{2}^{1}\right|_{\alpha, \Gamma}
$$

This finally yields

$$
\left\|\boldsymbol{H}_{\Gamma}^{*} \boldsymbol{G}_{2}^{1}\right\|_{\beta, \Gamma} \leq\left(1+|\Gamma|^{\alpha-\beta}+c_{1}(\mathbf{s}(\mathbf{d}))^{\frac{1-\beta}{2 n}}+c_{2}(\mathbf{s}(\mathbf{d}))^{\frac{1-\beta}{2 n}}|\Gamma|^{\beta}\right)\left\|\boldsymbol{G}_{2}^{1}\right\|_{\alpha, \Gamma}
$$

which completes the proof.

Remark 2 Note that in the proof of both Theorems 1 and 2 we have used the d-summability of $\Gamma$ only to ensure the finiteness of some $(d+\varepsilon)$-sum. In the following section we will exploit this argument when the surface is assumed to be ( $\ell$ ) AD-regular.

\subsection{The case of an ( $\ell$ AD-regular boundary}

Theorem 3 Let $\Gamma$ be ( $\ell$ ) AD-regular. Then the Hilbert transform $\mathcal{H}_{\Gamma}$ is bounded from $\mathcal{C}^{0, \alpha}(\Gamma)$ into $\mathcal{C}^{0, \beta}(\Gamma)$, whenever $0<\beta<2 n \alpha+1-2 n<1$. Moreover,

$$
\left\|\mathcal{H}_{\Gamma}\right\| \leq 1+c_{1}\left[\mathcal{H}^{2 n-1}(\Gamma)\right]^{\frac{\alpha-\beta}{2 n-1}}+c_{2}\left[\mathcal{H}^{2 n-1}(\Gamma)\right]^{\frac{1-\beta}{2 n}}+c_{3}\left[\mathcal{H}^{2 n-1}(\Gamma)\right]^{\frac{2 n-1+\beta}{2 n(2 n-1)}}
$$

where $c_{1}, c_{2}$ and $c_{3}$ only depend on $\alpha, \beta, n$ and on the constant $c_{\Gamma}$, introduced in (1).

Proof.

Take $p=\frac{2 n}{1-\beta}$, then, by assumption, $1-p(1-\alpha)>0$, whence Lemma 3 implies that the corresponding $(2 n-p(1-\alpha))$-sum is finite. According to Remark 2 , the estimate (6) then follows similarly as in the proof of Theorem 2 . The proof is further completed by the following observations:

(i) from (1), it follows that $|\Gamma| \leq c_{\Gamma}^{-\frac{1}{2 n-1}}\left[\mathcal{H}^{2 n-1}(\Gamma)\right]^{\frac{1}{2 n-1}}$;

(ii) from Remark 1 , taking $\varepsilon=1-p(1-\alpha)$, it follows that $\mathbf{s}(p(\alpha-\beta)) \leq c \frac{\mathcal{H}^{2 n-1}(\Gamma)}{1-p(1-\alpha)}$. 


\section{Acknowledgments}

This paper was written during a scientific stay of the second author at the Clifford Research Group of the Department of Mathematical Analysis at Ghent University. He wishes to thank the members of the Clifford Research Group for their kind hospitality during this stay.

\section{References}

[1] R. Abreu Blaya, J. Bory Reyes, F. Brackx, H. De Schepper, F. Sommen, A Hilbert transform for matrix functions on fractal domains, submitted.

[2] R. Abreu Blaya, J. Bory Reyes, F. Brackx, H. De Schepper, F. Sommen, A Hermitean Cauchy formula on a domain with fractal boundary, J. Math. Anal. Appl. (2010).

[3] R. Abreu Blaya, J. Bory Reyes, F. Brackx, B. De Knock, H. De Schepper, D. Peña Peña, F. Sommen, Hermitean Cauchy integral decomposition of continuous functions on hypersurfaces, Bound. Value Probl. 2008 (2008), Article ID 425256.

[4] R. Abreu Blaya, J. Bory Reyes, F. Brackx, H. De Schepper, Hermitean Téodorescu transform decomposition of continuous matrix functions on fractal hypersurfaces, Bound. Value Probl. 2010 (2010), Article ID 791358.

[5] R. Abreu Blaya, J. Bory Reyes, D. Peña Peña, F. Sommen, A boundary value problem for Hermitian monogenic functions, Bound. Value Probl. 2008 (2008), Article ID 385874.

[6] R. Abreu Blaya, J. Bory Reyes, T. Moreno García, Cauchy Transform on non-rectifiable surfaces in Clifford Analysis, J. Math. Anal. Appl. 339 (2008), 31-44.

[7] S. Bernstein, L. Lanzani, Szegö projections for Hardy spaces of monogenic functions and applications, IJMMS 29(10) (2002), 613624.

[8] F. Brackx, J. Bureš, H. De Schepper, D. Eelbode, F. Sommen, V. Souček, Fundaments of Hermitean Clifford analysis - Part I: Complex structure, Complex Anal. Oper. Theory 1(3) (2007), 341-365.

[9] F. Brackx, J. Bureš, H. De Schepper, D. Eelbode, F. Sommen, V. Souček, Fundaments of Hermitean Clifford analysis - Part II: Splitting of h-monogenic equations, Complex Var. Elliptic Equ. 52(10-11) (2007), 1063-1079.

[10] F. Brackx, B. De Knock, H. De Schepper, A matrix Hilbert transform in Hermitean Clifford analysis, J. Math. Anal. Appl. 344 (2008), 1068-1078.

[11] F. Brackx, B. De Knock, H. De Schepper, F. Sommen, On Cauchy and Martinelli-Bochner integral formulae in Hermitean Clifford analysis, Bull Braz Math Soc. 40(3) (2009), 395-416.

[12] F. Brackx, R. Delanghe, F. Sommen, Clifford analysis, Research Notes in Mathematics 76, Pitman (Advanced Publishing Program), Boston, 1982.

[13] F. Brackx, H. De Schepper, D. Eelbode, A new Hilbert transform on the unit sphere in $\mathbb{R}^{m}$, Comp. Var. Ell. Equ. 51(5-6), 2006, 453-462.

[14] F. Brackx, H. De Schepper, D. Eelbode and V. Souček, The Howe dual pair in Hermitean Clifford analysis, Rev. Mat. Iberoamericana 26(2) (2010), 449-479.

[15] F. Brackx, H. De Schepper, F. Sommen, The Hermitean Clifford analysis toolbox, Adv. Appl. Cliff. Alg. 18(3-4) (2008), 451-487.

[16] F. Brackx, N. Van Acker, $H^{p}$ spaces of monogenic functions. In: A. Micali et al. (eds.), Clifford Algebras and their Applications in Mathematical Physics, Kluwer Academic Publishers (Dordrecht, 1992), 177-188. 
[17] F. Colombo, I. Sabadini, F. Sommen, D.C. Struppa, Analysis of Dirac Systems and Computational Algebra, Birkhäuser, Boston, 2004.

[18] R. Delanghe, Some remarks on the principal value kernel in $\mathbb{R}^{m}$, Comp. Var.: Theory 8 Applic. 47 (2002), 653-662.

[19] R. Delanghe, On the Hardy spaces of harmonic and monogenic functions in the unit ball of $\mathbb{R}^{m+1}$. In: Acoustics, mechanics and the related topics of mathematical analysis, World Scientific Publishing (River Edge, New Jersey, 2002), 137142.

[20] R. Delanghe, On Some Properties of the Hilbert Transform in Euclidean Space, Bull. Belg. Math. Soc. - Simon Stevin 11 (2004), 163-180.

[21] R. Delanghe, F. Sommen, V. Souček, Clifford Algebra and Spinor-Valued Functions, Kluwer Academic Publishers, Dordrecht, 1992.

[22] K.J. Falconer, The geometry of fractal sets, Cambridge Tracts in Mathematics 85, Cambridge University Press, Cambridge, 1986.

[23] J. Feder, Fractals (With a foreword by Benoit B. Mandelbrot), Physics of Solids and Liquids, Plenum Press, New York, 1988.

[24] H. Federer, Geometric measure theory, Die Grundlehren der mathematischen Wissenschaften 153, Springer Verlag, New York Inc., New York, 1969.

[25] K. Gürlebeck, K. Habetha, W. Sprössig, Holomorphic functions in the plane and ndimensional space, Birkhäuser Verlag, Basel, 2008.

[26] J. Gilbert, M. Murray, Clifford Algebras and Dirac Operators in Harmonic Analysis, Cambridge University Press, Cambridge, 1991.

[27] J. Harrison, A. Norton, The Gauss-Green theorem for fractal boundaries, Duke Mathematical Journal 67(3) (1992), 575-588.

[28] J. Horváth, Sur les fonctions conjuguées à plusieurs variables (French), Koninklijke Nederlandse Akademie van Wetenschappen, Proceedings Series A $\mathbf{5 6}=$ Indagationes Mathematicae 15 (1953), 17-29.

[29] V.V. Kravchenko, M.V. Shapiro, Integral Representations for Spatial Models of Mathematical Physics, Pitman Research Notes in Mathematics Series 351, Longman Scientific and Technical (Harlow, 1996).

[30] M.L. Lapidus, H. Maier, Hypothse de Riemann, cordes fractales vibrantes et conjecture de Weyl-Berry modifie (French), C.R. Acad. Sci. Paris Série I Math. 313(1) (1991), 19-24.

[31] C. Li, A. McIntosh, T. Qian, Clifford algebras, Fourier transforms and singular convolution operators on Lipschitz surfaces, Rev. Math. Iberoamer. 10, 1994, 665-721.

[32] C. Li, A. McIntosh, S. Semmes, Convolution singular integrals on Lipschitz surfaces, J. Amer. Math. Soc. 5, 1992, 455-481.

[33] P. Mattila, Geometry of sets and measures in Euclidean spaces. Fractals and rectifiability, Cambridge Studies in Advanced Mathematics 44, Cambridge University Press, 1995.

[34] A. McIntosh, Fourier theory, singular integrals, and harmonic functions on Lipschitz domains. In: J. Ryan (ed.), Clifford Algebras in Analysis and Related Topics, Studies in Advanced Mathematics, CRC Press, Boca Raton, 1996, 33-87.

[35] M. Mitrea, Clifford Wavelets, Singular Integrals and Hardy Spaces, Lecture Notes in Mathematics 1575, Springer-Verlag (Berlin, 1994).

[36] R. Rocha-Chávez, M. Shapiro, F. Sommen, Integral theorems for functions and differential forms in $\mathbb{C}^{m}$, Research Notes in Mathematics 428, Chapman \& Hall/CRC, Boca Raton, 2002. 
[37] J. Ryan, Complexified Clifford analysis, Comp. Var. Theory Applic. 1(1) (1982/83), 119-149.

[38] I. Sabadini, F. Sommen, Hermitian Clifford analysis and resolutions, Math. Methods Appl. Sci. 25(16-18) (2002), 1395-1413.

[39] M.V. Shapiro, N.L. Vasilevski, Quaternionic $\psi$-holomorphic functions, singular integral operators and boundary value problems, Parts I and II, Complex Variables: Theory and Application 27, 1995, 14-46 and 67-96.

[40] E.M. Stein, Singular Integrals and Differentiability Properties of Functions, Princeton Math. Ser. 30, Princeton Univ. Press, Princeton, 1970. 